\title{
Cytokine Filter Application in COVID-19 Patients; Island of Hope for Crash and Burn Patients or Future Solution for All Septic Acute Respiratory Distress Syndrome (ARDS) Patients
}

\author{
Ali Ghodsizad ${ }^{1}$ \\ ${ }^{1}$ University of Miami
}

June 7, 2021

Cytokine Filter Application in COVID-19 Patients; Island of Hope for Crash and Burn Patients or Future Solution for All Septic Acute Respiratory Distress Syndrome (ARDS) Patients

\author{
Ali Ghodsizad MD, PhD, FACC, FETCS, FACS
}

The COVID-19 pandemic crisis certainly has challenged the scientific community as well as entire world. While incidence numbers have decreased following expedited vaccination and precautions, still some patients present with COVID 19 related pneumonia and ARDS requiring Veno-Venous Extracorporeal Membrane Oxygenation (VV ECMO) support to survive.

In COVID-19 patients a cytokine release syndrome concomitant with ARDS can lead to overwhelming clinical scenario. Geraci and colleagues report on their single center feasibility study looking at application of the Cytosorb $^{\mathrm{TM}}$ hemadsorption device which was used as a parallel circuit within the VV ECMO circuit.

The authors give evidence for safety and feasibility of the Cytosorb ${ }^{\mathrm{TM}}$ hemadsorption device use in 10 patients with COVID-19 related ARDS in combination with VV ECMO. They show a reduction of inflammatory markers and cytokines following hemadsorption treatment. The cytokine storm can cause a critical clinical picture of septic shock. Only under high vasopressor and inotropic support end organ perfusion can be maintained. The required invasive pressure ventilation with high PEEP and peak pressure can decrease the intrathoracic venous return further and contributes more to the shock physiology (1). We have to look at inspiring results from current single center experience carefully understanding the evolving nature of COVID-19 related ARDS. Other groups have used plasmapheresis and CVVH modifications in COVID-19 cases. Dominik et al have shown a significant benefit only using hemadsorption comparing to other used protocols (2).

We have shown successful application of somatic stem cells in COVID-19 patients on VV ECMO at our center. We could observe a reduction of inflammatory markers following somatic stem cell application (3). COVID 19 ARDS patients who required VV ECMO support, underwent a Pulmonary Artery (PA)-catheter placement and allogenic human stem cell injection into the PA using the PA-catheter as part of our expanded access protocol $(3,4)$.

Brouwer and colleagues, another group working with hemadsorption, have actually shown reduced survival in patients undergoing hemadsorption therapy (5). Geraci and colleagues describe their overall VV ECMO survival for COVID 19 related respiratory failure to be $>90 \%$. Others including our center have experienced a much lower survival in that patient population. So patient selection clearly is a key point. The results presented by Geraci and colleagues have to be taken as a pioneering step, which can help in ARDS and septic clinical scenarios with different pathology in future. 


\section{References:}

Grant AA et al, A Weaning Protocol for Venovenous Extracorporeal Membrane Oxygenation With a Review of the Literature.

Artif Organs. 2018 Jun;42(6):605-610

1. Dominik et al, Similarities, Differences, and Potential Synergies in the Mechanism of Action of Albumin Dialysis Using the MARS Albumin Dialysis Device and the CytoSorb Hemoperfusion Device in the Treatment of Liver Failure. Blood Purif. 2021;50(1):119-128. doi: 10.1159/000508810. Epub 2020 Jul 2 .

2. Kaushal et al, Intravenous mesenchymal stem cells in ECMO patients with severe COVID 19 ARDS, Med $R_{X}$ IV, 2020

3. A Ghodsizad et al, Transplanted human cord blood-derived unrestricted somatic stem cells improve left-ventricular function and prevent left-ventricular dilation and scar formation after acute myocardial infarction. Heart. 2009 Jan;95(1):27-35. doi: 10.1136/hrt.2007.139329. Epub 2008 Jun 2

4. Brouwer et al, Hemoadsorption with CytoSorb shows a decreased observed versus expected 28-day all-cause mortality in ICU patients with septic shock: a propensity-score-weighted retrospective study. Critical Care. 2019 Sep 18;23(1):317. 